\title{
Silica Embedded Metal Hydrides
}

by

L. K. Heung

Westinghouse Savannah River Company

Savannah River Site

Aiken, South Carolina 29808

G. G. Wicks

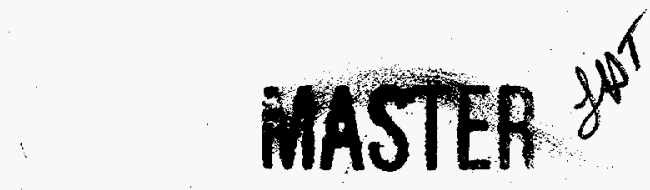

A document prepared for INTERNATIONAL SYMPOSIUM ON METAL HYDRIDE SYSTEMS, FUNDAMENTALS, AND APPLICATIONS at Hangzhou, , China from 10/4/98 - 10/9/98.

DOE Contract No. DE-AC09-96SR18500

This paper was prepared in connection with work done under the above contract number with the U.S. Department of Energy. By acceptance of this paper, the publisher and/or recipient acknowledges the U.S. Government's right to retain a nonexclusive, royalty-free license in and to any copyright covering this paper, along with the right to reproduce and to authorize others to reproduce all or part of the copyrighted paper. 


\section{DISCLAIMER}

This report was prepared as an account of work sponsored by an agency of the United States Government. Neither the United States Government nor any agency thereof, nor any of their employees, makes any warranty, express or implied, or assumes any legal liability or responsibility for the accuracy, completeness, or usefulness of any information, apparatus, product, or process disclosed, or represents that its use would not infringe privately owned rights. Reference herein to any specific commercial product, process, or service by trade name, trademark, manufacturer, or otherwise does not necessarily constitute or imply its endorsement, recommendation, or favoring by the United States Government or any agency thereof. The views and opinions of authors expressed herein do not necessarily state or reflect those of the United States Government or any agency thereof.

This report has been reproduced directly from the best available copy.

Available to DOE and DOE contractors from the Office of Scientific and Technical Information, P. O. Box 62, Oak Ridge, TN 37831; prices available from (423) 576-8401.

Available to the public from the National Technical Information Service, U. S. Department of Commerce, 5285. Port Royal Road, Springfield, VA 22161. 


\section{DISCLAIMER}

Portions of this document may be illegible in electronic image products. Images are produced from the best available original document. 


\title{
Silica Embedded Metal Hydrides
}

\author{
L. K. Heung, G. G. Wicks \\ Westinghouse Savannah River Company, 773-A, Savannah River Site \\ Aiken, SC 29808 USA
}

\begin{abstract}
A method to produce silica embedded metal hydride was developed. The product is a composite in which metal hydride particles are embedded in a matrix of silica. The silica matrix is highly porous. Hydrogen gas can easily reach the embedded metal hydride particles. The pores are small so that the metal hydride particles cannot leave the matrix. The porous matrix also protects the metal hydride particles from larger and reactive molecules such as oxygen, since the larger gas molecules cannot pass through the small pores easily. Tests show that granules of this composite can absorb hydrogen readily and withstand many cycles without producing fines.
\end{abstract}

\section{INTRODUCTION}

Metals and metal alloys, which can react with hydrogen reversibly to form metal hydrides, have long been studied for hydrogen separation applications [1]. Many of these metal hydrides can absorb then desorb large amounts of hydrogen under moderate change of temperature and/or pressure. A simple temperature or pressure swing absorption process using metal hydrides can be designed to recover hydrogen from gas mixtures economically, if not for a few engineering and chemical problems.

Metal hydrides expand upon hydrogen absorption and contract upon hydrogen desorption. This volume change associated with each absorption/desorption cycle creates engineering problems. It generates a cyclic internal stress on the material that breaks up the metal hydride particles to fines. The fines, being micron size particles, are not suitable for use in large separation columns, because they restrict gas flow and can clog filters. Furthermore, they settle down in the bottom of a packed column and can generate enough stress on the column wall to cause bulging and even splitting of the column.

Metal hydrides are reactive to oxygen. Exposure to low concentrations of oxygen causes gradual loss of hydrogen capacity. Exposure to high concentrations of oxygen can render the metal hydride inactive to hydrogen. Metal hydrides can also be poisoned by certain impurities, such as carbon monoxide, to become inactive to hydrogen.

Therefore, before metal hydrides can be successfully applied in large hydrogen separation processes, they must be converted into a stable form that will not break down to fines. They also must be resistant to oxygen and other impurities.

Attempts have been made to convert metal hydride powders to stable packing materials in the form of pellets or granules. Metal hydride powders have been mixed with other metal powders such as copper, aluminum or nickel. This mixture is formed into pellets by compression and then sintered with heat under vacuum, inert gas or hydrogen. The resulted pellet is mechanically strong and can withstand many absorption cycles without breaking down. However, the permeation of hydrogen through the sintered pellet is slow [2]. When the amount of sintering is reduced to increase porosity and permeation rate, the pellets are weakened and the pores are larger than the metal hydride fines. The fines eventually work their way out of the pellet.

Better ways to make metal hydride based packing materials are still needed. 


\section{GENNERAL APPROACH TO THE PROBLEM}

Breaking down of metal hydride particles to micron size fines is an inherent problem common to metal hydrides. The question is: can one embed metal hydride fines in a porous matrix which will not break up after many absorption cycles. The porosity must be very high so that hydrogen gas can reach the metal hydride particles easily. But the pores must be smaller than the metal hydride fines so that the fines cannot escape from the matrix. Furthermore, the pores should be sufficiently small so that gas molecules larger than hydrogen are excluded.

It is well known that highly porous silica gels can be made via the sol-gel process [3]. The pore size of the dried gels can be varied from less than $30 \AA$ to larger than $100 \AA(0.01$ micron) dependent on fabrication conditions. It is also known that similar highly porous silica material can also be produced from fumed silica products. The pores in this porous silica can be 10 times or more smaller than the smallest metal hydride particles. It appears that silica gel is a perfect porous matrix to embed and trap the metal hydride fines. The high porosity of these gels provides plenty of paths for hydrogen gas to reach the embedded metal hydride particles. The pore size can also be controlled to screen out impurities other than hydrogen.

The process to embed metal hydride particles in porous silica was studied and the results are presented in this paper.

\section{EXPERIMENTAL DETAILS}

\subsection{Sample Preparation}

\section{Making Metal Hydride Powders}

Commercially produced $\mathrm{LaNi}_{4.25} \mathrm{Al}_{0.75}$ in the form of millimeter size particles is first activated by exposing to 5 atm hydrogen gas at room temperature, and then subjected to repeated hydriding and dehydriding. After about 20 cycles, most of the particles will break up to powders small enough to go through a 45 micron sieve. These fresh metal powders must not be exposed to open air in one single step, because such an air exposure will cause too much oxidation of the metal and render the material useless. They must be exposed to limited air gradually over several days, so that a thin layer of oxide can slowly form on the surface of the metal particles. This thin oxide layer protects the metal particle from further oxidation. The particles are then become stable and can be handled in open air. After sieving through a 45 micron sieve, the metal hydride powders are saved for later use.

\section{Porous Silica Formation}

Porous silica may be produced using a sol-gel process. The process starts with the hydrolysis of an organo metallic compound. The hydrolyzed compound is polymerized via water and alcohol condensation. A dry product is produced after the water and alcohol are removed. Thermal treatment following the drying step is sometimes used to further modify the structure of the final product. The reactions involved are described as follows:

Hydrolysis:

$(\mathrm{OR})_{3} \mathrm{M}-\mathrm{OR}+\mathrm{H}_{2} \mathrm{O}==(\mathrm{OR})_{3} \mathrm{M}-\mathrm{OH}+\mathrm{ROH}$ 
Water condensation:

$(\mathrm{OR})_{3} \mathrm{M}-\mathrm{OH}+(\mathrm{OR})_{3} \mathrm{Si}-\mathrm{OR}==(\mathrm{OR})_{3} \mathrm{M}-\mathrm{O}-\mathrm{M}(\mathrm{OR})_{3}+\mathrm{H}_{2} \mathrm{O}$

Alcohol condensation:

$(\mathrm{OR})_{3} \mathrm{M}-\mathrm{OH}+(\mathrm{OR})_{3} \mathrm{M}-\mathrm{OH}==(\mathrm{OR})_{3} \mathrm{M}-\mathrm{O}-\mathrm{M}(\mathrm{OR})_{3}+\mathrm{ROH}$

Final products:

11

[ -M-O-M-O- ] $, \mathrm{H}_{2} \mathrm{O}, \mathrm{ROH}$

11

where $\mathrm{M}$ is a metallic element such as $\mathrm{Si}, \mathrm{Al}$ and $\mathrm{Ti}$, and $\mathrm{R}$ is an alkyl group $\left(-\mathrm{C}_{\mathbf{x}} \mathrm{H}_{(2 x+1)}\right)$.

Other than the above sol-gel approach, porous silica can also be produced using fumed silica. Commercially available fumed silica is produced by the hydrolysis of silicon tetrachloride vapor in a flame of hydrogen and oxygen. In the combustion process molten spheres of silica are formed. The diameters of these spheres are in the order of nanometers. They collide and fuse with one another to form branched, three-dimensional, chain-like aggregates. Fumed silica can polymerize via the hydrolysis process when blended with water, to form a highly porous product.

\section{Embedding Metal Hydride In Silica}

Metal hydride powders are embedded in a porous silica matrix by using two different methods. The first method uses a sol-gel to produce the matrix. The second method uses the fumed silica. Both methods can encapsulate metal hydride particles at ambient or low temperatures, which have no adverse effect on the integrity or properties of the particles.

Sol-Gel Method: The sol-gel method to embed the metal hydride particles involves the following steps. Solution A is prepared by adding ethanol to water while stirring. The ethanol to water ratio is two to five part water to one part water. Acidity of the solution is adjusted by adding $\mathrm{HCl}$ until the $\mathrm{pH}$ value is in the range of 1 to 2.5 . Solution $\mathrm{B}$ is prepared by mixing ethanol into tetraethyl orthosilicate (TEOS) in the ratio of one part ethanol to two part TEOS. Solution A is added to solution B slowly while stirring continuously to form a sol. To the sol a measured amount of the metal hydride powders prepared earlier is added. The mixture is stirred to keep the metal hydride particles dispersed uniformly throughout the sol. The mixing continuous until the sol turns into gel and the metal hydride particles become suspended in the gel. The gel is then allowed to mature for several days. When the polymerization is complete, the gel becomes a solid phase submerged in the liquid water and ethanol. The liquid is evaporated at room temperature, followed by evacuation at temperature of up to about $600{ }^{\circ} \mathrm{C}$. After drying, large pieces of silica embedded metal hydride are formed. The large pieces are mechanically broken up to granules of $1-5 \mathrm{~mm}$ size for testing.

Fumed Silica Method: In this method, commercially available CAB-O-SIL ${ }^{\circledR}$ fumed silica from Cabot Corporation is used to embed the metal hydride particles. The fumed silica, in the form of a light and fluffy powder, is added and blended into water a little at a time. A water-like sol is formed. The mole ratio of water to fumed silica is between 10 to 30 . While stirring 
continuously, a measured amount of metal hydride powders is added, also in small steps. In the end, a uniform, thick, watery mixture is formed. The mixture is poured quickly into a tray and spread out to solidify. The solid is dried in air and then broken into granules of 1-5 mm size for testing. Micrographs of silica embedded metal hydride particles are shown in Figure 1.

\section{PERFORMANCE TESTS}

\subsection{Test Apparatus and Procedure}

The test apparatus is shown schematically in Figure 2. It is a manifold fabricated from $1 / 4$ inch $(0.635 \mathrm{~cm})$ stainless steel tubes, metal bellows sealed Nupro ${ }^{\circledR}$ valves and Cajon ${ }^{\circledR}$ fittings. The sample container is assembled from stainless steel Cajon ${ }^{\circledR}$ fittings. The volumes of the tubing sections between valves are calibrated and are used to measure the volumes of hydrogen to be absorbed by the sample. The pressure is measured by MKS Barratron ${ }^{\circledR}$ pressure transducers $(10,000$ torr range). A crucible furnace with a temperature controller is used to control the temperature of the sample.

A typical test involves several steps. The sample is first evacuated at room temperature or at $150{ }^{\circ} \mathrm{C}$ depending on the test. Hydrogen is absorbed by the sample at room temperature, and desorbed at $150^{\circ} \mathrm{C}$. Five absorption/desorption cycles are normally conducted on a sample. The amount of hydrogen absorbed each time is calculated from the pressure changes in the known volumes. For some samples, after the fifth desorption, a valve separating room air from the sample is opened for about 5 minutes to let room air into the sample. A sixth absorption is then conducted after the air is evacuated. After the last desorption, the sample is removed from the container and its physical condition is compared with the original.

\subsection{Test Results}

The performances of the samples are compared by ease of activation, hydrogen capacity, effect of absorption cycles, and physical changes caused by hydrogen absorption and desorption.

Activation: Before a metal hydride can absorb hydrogen readily, it usually requires an activation step. The activation step normally involves baking out the sample at vacuum and then exposing the sample to hydrogen at several times its equilibrium pressure. For the metal hydride used in this work, the bake out temperature was $150^{\circ} \mathrm{C}$ and the time was 2 hours. The hydrogen pressure was about 3000 torr ( $4 \mathrm{~atm}$ ). Sample size was between 0.5 to $1 \mathrm{gram}$.

The activation behavior of a silica embedded metal hydride sample is shown in Figure 3 . The first curve was generated from a test to see if the sample absorbs hydrogen without bake-out: The sample was first evacuated at room temperature for 2 hours. It was then opened to a calibrated volume filled with 4000 torr of hydrogen. The first pressure drop was due to the volume expansion of the sample container. The sample did not absorb any hydrogen after two hours (only ten minutes are shown in the figure). The second curve was produced when the sample absorbed hydrogen for the first time after it was baked out at $150{ }^{\circ} \mathrm{C}$. The first data point was mostly due to volume expansion; but the pressure continued to drop indicating the hydrogen was absorbed. In about 4 minutes the sample was saturated with hydrogen to a hydrogen content of approximately 0.7 hydrogen atom per one metal atom $(\mathrm{H} / \mathrm{M})$. The third curve shows the second hydrogen absorption of the sample after it was desorbed at $150{ }^{\circ} \mathrm{C}$ under evacuation. The 
sample absorbed hydrogen immediately and reached saturation in about 2 minutes, indicating the sample was fully activated.

The activation behavior of a silica embedded metal hydride sample is compared with a pure metal hydride sample in Figure 4. Both samples were baked out at $150{ }^{\circ} \mathrm{C}$ under evacuation for 2 hours before they were exposed to hydrogen. The first curve in Figure 4 belongs to the pure metal hydride. It took about 20 minutes before the sample began to absorb hydrogen, and took another 30 minutes before it was saturated with hydrogen. The second curve belongs to the silica embedded metal hydride. The sample began to absorb hydrogen almost immediately and become saturated in less than five minutes. The composite sample was much easier to activate than the pure metal hydride.

Hydrogen Capacity: For this work, the hydrogen capacity of a sample was measured by the amount of hydrogen it could absorb at room temperature. The sample was exposed to hydrogen at room temperature until it was saturated with hydrogen at pressures between 500 to 3000 torr. The hydrogen capacity is expressed as $\mathrm{H} / \mathrm{M}$, the number of hydrogen atoms per atom of metal hydride in the sample. This value is only slightly affected by the final hydrogen pressure, because the final pressure is more than 10 times the equilibrium plateau pressure. The data shown in Table 1 indicate that the embedded metal hydride samples have about the same hydrogen capacity as that of the pure metal hydride.

Particle Breakdown and Cycling Effect: As discussed in the earlier section, one absorption/desorption cycle can break the metal hydride particles. All the silica embedded metal hydride samples, with hydride content of up to $70 \%$ by weight, did not show any break down after 5 absorption/desorption. To see the effect of increased number of cycles, an embedded sample was tested for 100 absorption/desorption cycles. The metal hydride content in this sample was $27.7 \mathrm{wt} \%$. For each cycle, the sample is first saturated with hydrogen at approximately 2,000 torr pressure at room temperature, and then the hydrogen is desorbed at $150{ }^{\circ} \mathrm{C}$ under vacuum. The amounts of hydrogen absorbed and desorbed in each cycle are about $0.77 \mathrm{H} / \mathrm{M}$ (atom ratio of hydrogen to metal). After 100 cycles the sample was taken out for visual and SEM examination. There was no sign of any particle breakdown. The particles in the sample looked the same as before cycling. In Figure 5a, the effects of only two hydrogen absorption cycles on metal hydride particles alone are shown and the fines produced are of micron size. In Figure $\mathbf{5 b}$ and $\mathbf{5 c}$, the effect of 100 hydrogen absorption cycles on a fumed silica embedded metal hydride is shown before and after the cycling. Note that there is practically no difference between the two and no fines are produced. The 100 cycles did not break down the particles.

Heat Treatment Effect: Samples of the silica embedded metal hydride were heated under vacuum at temperatures ranging from $200{ }^{\circ} \mathrm{C}$ to $600{ }^{\circ} \mathrm{C}$ for two hours. Their hydrogen absorption performances were measured after the heat treatment. The results are shown in Table 2. The data indicated a continuous decrease of hydrogen capacity as the temperature increased from 200 to $600{ }^{\circ} \mathrm{C}$. The sample produced with the fumed silica method showed less capacity decrease that the ones produced with the sol-gel method. The samples treated at $400^{\circ} \mathrm{C}$ showed improved resistance to air. They absorbed hydrogen readily without the usual bake-out step. These results suggest that heat treatment reduced the pore size of the silica matrix. Some of the metal hydride particles might have been completely masked by solid silica and become unavailable for hydrogen absorption. The pore size reduction did show a positive effect on preventing the oxygen in air from deactivating the metal hydride. 


\section{CONCLUSIONS}

Metal hydride powders can be embedded in a matrix of porous silica via a sol-gel process or using a fumed silica method. The resulting product, a silica embedded metal hydride, can be made into granules of desired size suitable for use in hydrogen separation columns. These granules are highly porous and permit hydrogen gas to reach the embedded metal hydride powders easily. Yet the pores are so fine that the metal hydride powders are effectively trapped inside the matrix. The fine pores can also restrict the passage of the larger molecules of oxygen and other harmful impurities, and therefore provide protection to the metal hydride powders from these impurities. The granules of this silica embedded metal hydride are fracture resistant and can withstand many absorption/desorption cycles without generating fines.

\section{References}

1. P. D. Goodell, W. J. Rebello and M. R. Ally, Final Report DOE/ID/12520 for Contract No. DE-AC07-84ID12520 with U. S. DOE, 1986.

2. E. Bershadsky, Y. Josephy and M. Ron, Metal-Hydrogen Systems Fundamentals and Applications, Stuttgart, 1988, volume II, p1373.

3. I. M. Thomas in Sol-gel technology for thin films, fibers, preforms, electronics, and specialty shapes, L. C. Klein (editor), 1988, p2. 
Table 1. Hydrogen Capacity Comparison.

\begin{tabular}{|lcc|}
\hline \multicolumn{1}{|c|}{ Sample } & $\begin{array}{c}\text { Wt \% Metal Hydride } \\
\left(\mathrm{LaNi}_{4} .25 \mathrm{Al}_{0.75)}\right.\end{array}$ & $\begin{array}{c}\text { Hydrogen Capacity } \\
\text { H/M }\end{array}$ \\
\hline $\begin{array}{l}\text { Pure LaNi4.25Al0.75 } \\
\text { Silica embedded LaNi4.25Al0.75 by the } \\
\text { sol-gel method }\end{array}$ & 100 & 0.76 \\
$\begin{array}{l}\text { Silica embedded LaNi4.25Al0.75 by the } \\
\text { fumed silica method }\end{array}$ & 23.8 & 0.76 \\
$\begin{array}{l}\text { Silica embedded LaNi4.25Al0.75 by the } \\
\text { fumed silica method } \\
\text { Silica embedded LaNi4.25Al0.75 by the } \\
\text { fumed silica method }\end{array}$ & 27.7 & 0.77 \\
\hline
\end{tabular}

Table 2. Heat Treatment Effect.

\begin{tabular}{|ccccc|}
\hline Sample & $\begin{array}{c}\text { Treatment } \\
\text { temperature } \\
{ }^{\circ} \mathrm{C}\end{array}$ & $\begin{array}{c}\text { Hydrogen capacity } \\
\mathrm{H} / \mathrm{M}\end{array}$ & $\begin{array}{c}\text { Hydrogen capacity } \\
\text { \% retained } \\
\text { before bake-out at } \\
150{ }^{\circ} \mathrm{C}\end{array}$ \\
\hline $\begin{array}{ccccc}\text { Silica embedded } \\
\text { LaNi4.25Al } 0.75 \text { by }\end{array}$ & $\begin{array}{c}\text { no treatment } \\
\text { the sol-gel method }\end{array}$ & 0.78 & 100 & 0 \\
\hline Silica embedded & $\mathbf{4 0 0}$ & 0.76 & 97 & 0 \\
LaNi4.25Al0.75 by & $\mathbf{4 0 0}$ & 0.31 & 40 & 0.31 \\
$\begin{array}{c}\text { the fumed silica } \\
\text { method }\end{array}$ & 600 & 0.66 & 92 & 0 \\
\hline
\end{tabular}



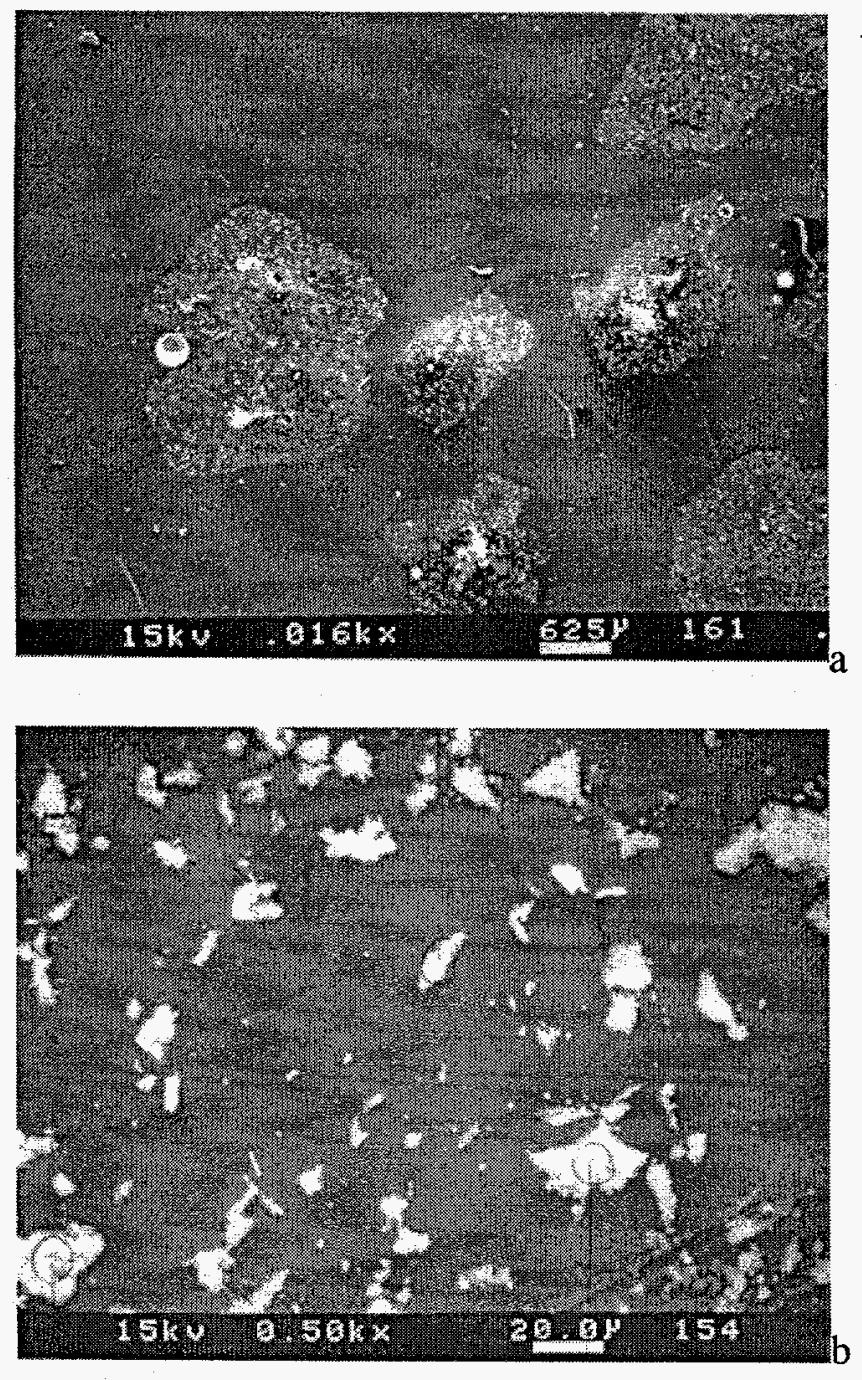

Figure 1. a) Particles of silica embedded metal hydride by fumed silica method (SEM 16X), b) polished surface of a particles of silica embedded metal hydride by fumed silica method (SEM 500X), white area: metal hydride, dark area: silica. 


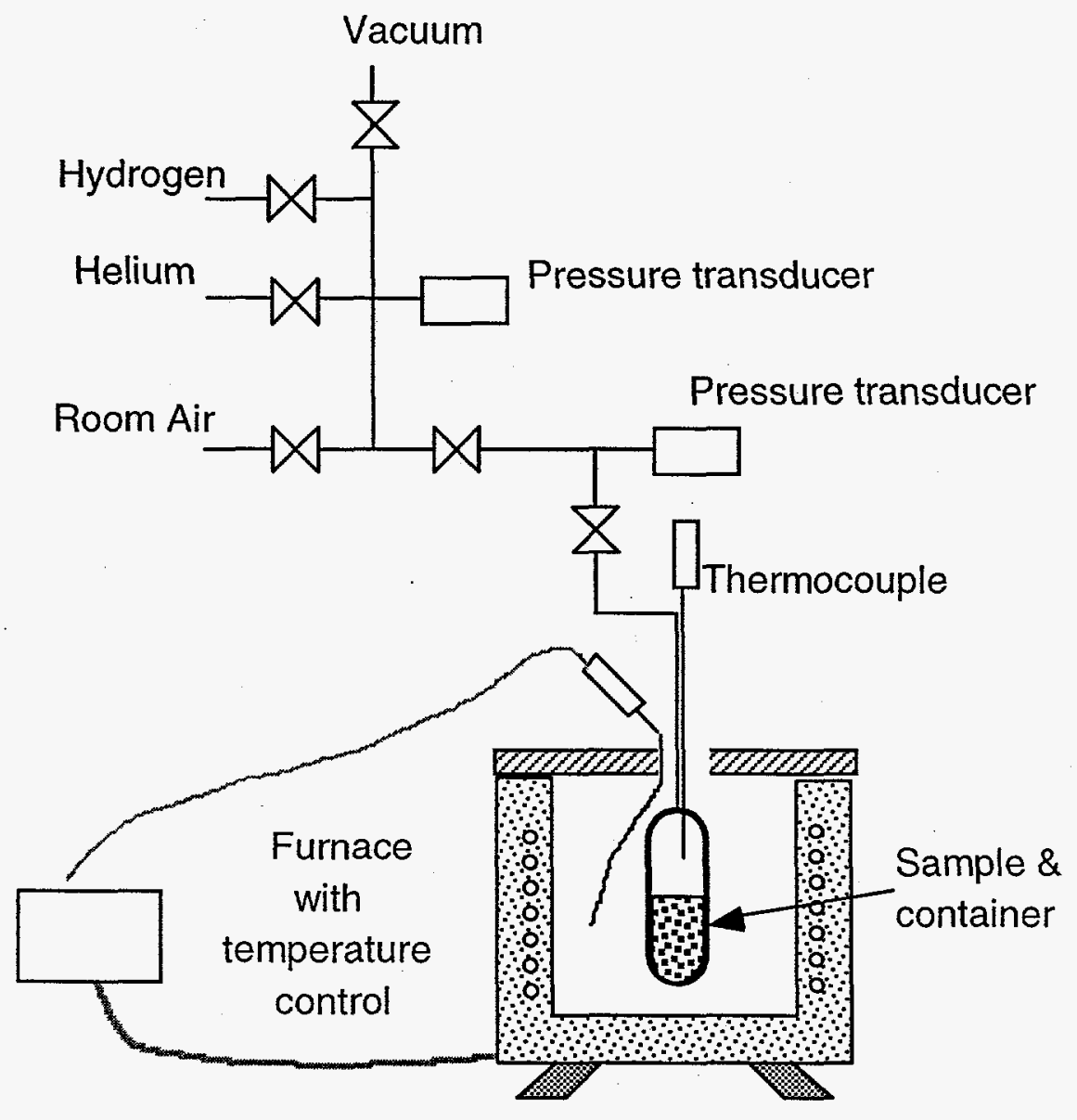

Figure 2. Test apparatus for hydrogen absorption/desorption tests. 


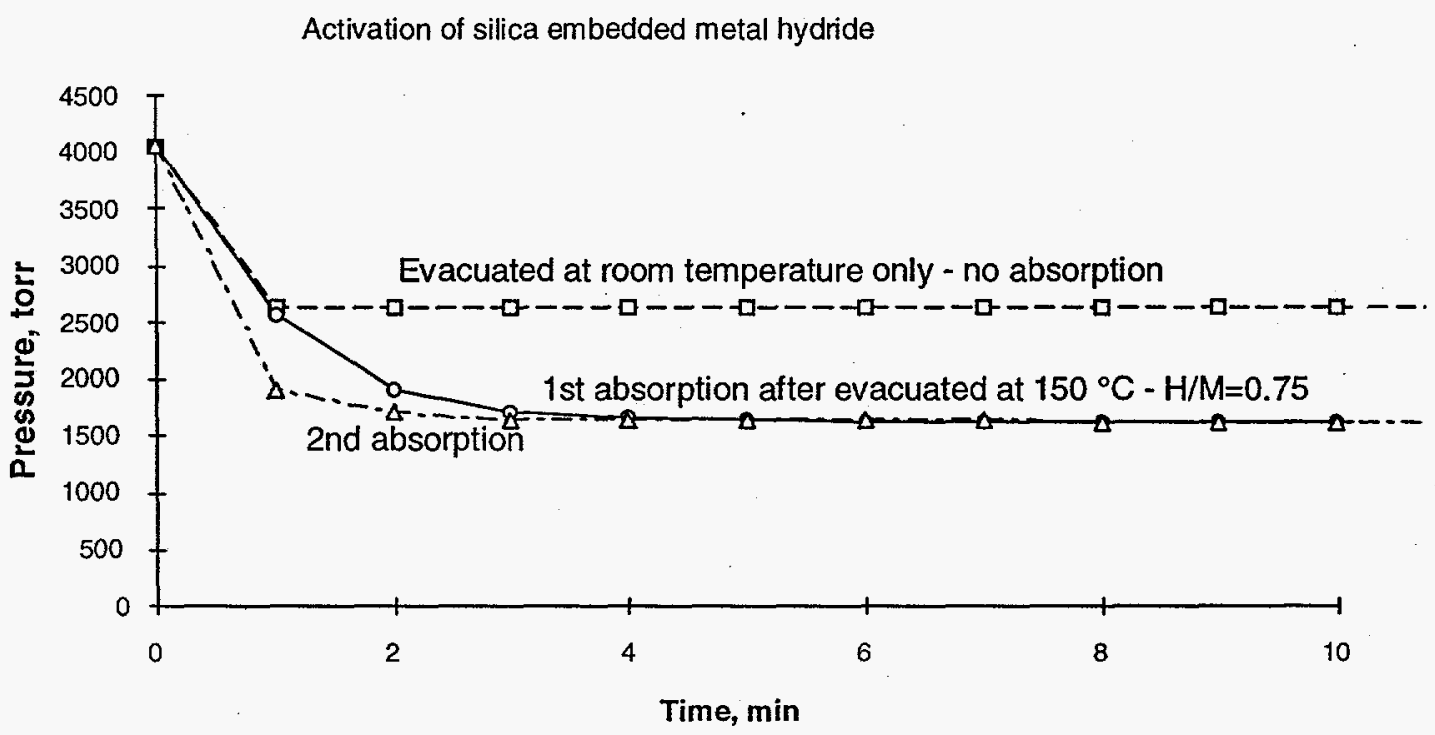

Figure 3. Hydrogen absorption of silica embedded $\mathrm{LaNi}_{4.25} \mathrm{Al}_{0.75}$.

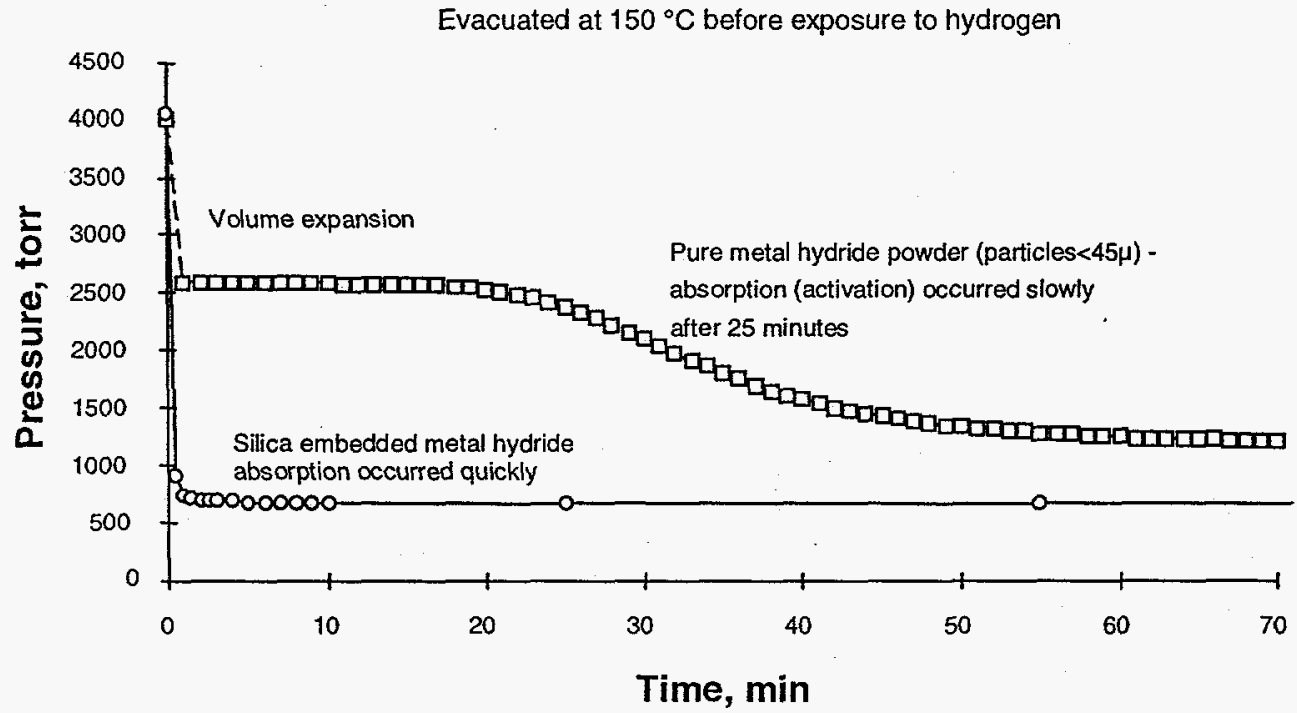

Figure 4. Hydrogen absorption comparison of pure and silica embedded $\mathrm{LaNi}_{4.25} \mathrm{Al}_{0.75}$. 

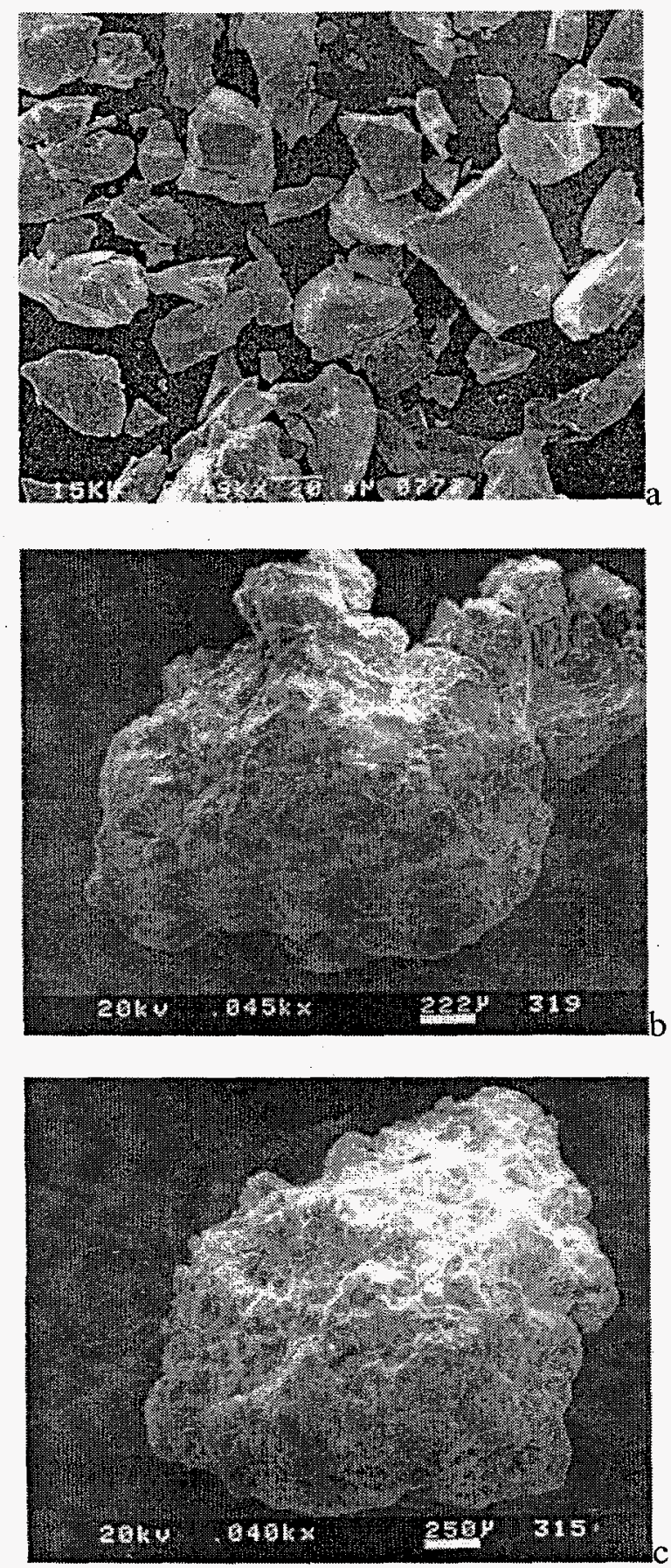

Figure 5. a) La-Ni-Al particle after 2 hydrogen absorption cycles (SEM 490X), particle broke down to micron size, b) silica embedded metal hydride particle (fumed silica method) before hydrogen absorption (SEM 45X), c) after 100 hydrogen absorption cycles, particle did not break down (SEM 40X). 